\title{
Essais
}

ESSAIS

Revue interdisciplinaire d'Humanités

$11 \mid 2017$

Fictions de l'identité

\section{Peut-on se passer de la notion d'identité ? La contribution de Montaigne}

Sylvia Giocanti

\section{(2) OpenEdition}

1 Journals

Édition électronique

URL : http://journals.openedition.org/essais/3294

DOI : $10.4000 /$ essais.3294

ISSN : 2276-0970

Éditeur

École doctorale Montaigne Humanités

Édition imprimée

Date de publication : 15 mai 2017

Pagination : 29-38

ISBN : 979-10-97024-01-7

ISSN : 2417-4211

\section{Référence électronique}

Sylvia Giocanti, «Peut-on se passer de la notion d'identité ? La contribution de Montaigne », Essais [En ligne], 11 | 2017, mis en ligne le 14 octobre 2020, consulté le 25 octobre 2020. URL : http://

journals.openedition.org/essais/3294; DOI : https://doi.org/10.4000/essais.3294 


\section{Peut-on se passer de la notion d'identité ? \\ La contribution de Montaigne}

\section{Sylvia Giocanti}

\section{L'homme sans identité spécifique}

Quel est l'apport de Montaigne dans le questionnement de l'identité ?

Montaigne est le philosophe qui soutient à la fois que "nous n'avons aucune communication à l'estre ${ }^{1}$ ", c'est-à-dire que nous n'avons pas accès à l'essence, à ce qui est un, identique à soi et que "chaque homme porte la forme entiere de l'humaine condition ${ }^{2}$ ", déclaration qui pourrait laisser entendre que tout individu a gravé en lui l'essence de l'espèce à laquelle il appartient et par laquelle il s'authentifie.

La conjonction de ces deux énoncés, qui peut paraître contradictoire, constitue en réalité une invitation à explorer la forme ou identité de l'homme en un autre sens qu'une détermination a priori qu'il faudrait connaître comme une nature (type) à réaliser. Il s'agit en effet pour Montaigne de récuser la tradition philosophique (celle d'Aristote, ou des stoïciens) selon laquelle nous nous devons de réaliser dans les faits un être préalablement déterminé par la pensée dans sa perfection, comme un modèle vers lequel il faudrait tendre. Il ne s'agit plus de vivre à l'image de l'archer qui, en visant la cible, réalise un plan préalable. Ce modèle est inopérant pour celui qui fait l'expérience de lui-même comme constitué de lopins, de formes rapiécées qui ne s'organisent pas entre elles: "Nous sommes tous de lopins, et d'une contexture si informe et diverse, que chaque pièce, chaque moment, faict son jeu. Et se trouve autant de difference de nous à nous mesmes, que de nous à autruy. Magnam rem puta unum hominem agere ${ }^{3}$.

Montaigne, Essais, II, 12, Villey (éd.), PUF, Quadrige, 1992, p. 601.

Ibid., III, 2, p. 805.

3 Ibid., II, 1, p. 337. La citation de Sénèque, détournée de son sens originel, peut se traduire ainsi : Pense que c'est une grande chose de jouer toujours le même homme. 
La condition humaine se comprend alors comme l'ensemble des formes indéfinies que chacun peut revêtir tour à tour, parce qu'il n'est disposé à rien par nature, et que celui qui est devenu cordonnier aurait très bien pu devenir empereur. La déclaration «les ames des empereurs et des savatiers sont jettees à mesme moule ${ }^{4}$ " fait écho à " chaque homme porte la forme entiere de l'humaine condition » et en atteste la portée anthropologique et sociale - et non pas normative et métaphysique - contre ceux qui pourraient croire que certains individus seraient davantage que d'autres prédisposés à " tenir le haut du pavé ".

À ceux-là, il faut rappeler que ce qui constitue notre identité et notre valeur d'homme dans la société est le fait du hasard', du lieu, du temps, des circonstances, des coutumes, c'est-à-dire d'un ensemble de déterminations extérieures et contingentes qui ne dépendent pas intrinsèquement de nous, dans la mesure où elles sont reçues, adoptées au fil du temps. Ainsi, « la forme entière de l'humaine condition ", loin de renvoyer à une essence qu'il faudrait connaître et réaliser en nous pour être dignes de notre humanité, désigne un ensemble de conditions d'existence dont on fait tous l'expérience - ce en quoi nous sommes tous " de la commune sorte "- qui se décline selon une variété irréductible ${ }^{6}$, dans l'inachèvement, puisque les potentialités de formes de l'humaine condition sont inépuisables, qu'on ne peut en sonder les limites.

Chaque homme a donc en partage l'instabilité de sa position, qui lui fait traverser la vie, "mouvement inegal, irregulier et multiforme", dans une mutation permanente, "la constance mesme n'est[ant] pas autre chose qu'un branle plus languissant ${ }^{7}$ ". S'employer à servir la vie selon elle, comme se le propose Montaigne, signifie alors se tenir " en son assiette ", c'est-à-dire non pas demeurer là où Dieu nous a mis, comme le préconise Saint Paul dans l'Épître aux Corinthiens (I, 15), mais apprendre à se tenir dans le passage, dans le devenir, qui n'est pas comme dans la tradition augustinienne jugé inférieur à l'être en repos, stable et identique à lui-même.

L'expérience que nous faisons de nous-mêmes est en effet celle d'une mutabilité et hétérogénéité indépassables, si bien que l'identité à soi est perçue non pas comme une fin à atteindre, mais comme une qualité qui nous est d'abord étrangère dans la manière d'apparaître à soi-même : «Ce qu'on remarque pour rare au Roy de Macedoine Perseus, que son esprit, ne s'attachant à aucune condition, allait errant par tout genre de vie et representant des mœurs si essorées et si vagabondes qu'il n'estoit cogneu ny de luy ny d'autre quel

4 Ibid., II, 12, p. 476.

5 Ibid., II, 1, p. 337 : « Nous vivons par hasard».

6 Ibid., III, 13, p. 1065 : "Il n'est aucune qualité si universelle en cette image des choses que la diversité et varieté ». Cf. I, 26, p. 157, au sujet de notre "mere nature » : " une si generale et constante varieté".

7 Voir respectivement Essais, III, 3, p. 819 et III, 2, p. 805. 
homme ce fust, me semble à peu pres convenir à tout le monde ". Ce qui est familier pour tout à chacun, c'est la différence à soi qui rend méconnaissable à soi-même et aux autres, du moins si l'on est attentif à la manière dont on se manifeste à soi-même avant la cristallisation du moi sous les différentes étiquettes que la vie sociale nous oblige à endosser tour à tour, selon nos fonctions et selon les circonstances de notre existence.

On ne saurait donc être à soi sans commencer par constater que " moy à cette heure et moy tantost sommes bien deux" ", que l'étrangeté et la difformité de chacun est telle, qu'elle ne cesse de l'étonner au premier chef : «On s'apprivoise à toute estrangeté par l'usage et le temps; mais plus je me hante et me connois, plus ma difformité m'estonne, moins je m'entens en moy ${ }^{10}$ ». Nul ne peut sans se mentir à lui-même se familiariser avec ses différences à "soi ", au point de constater en soi l'identité d'une seule forme. Celui qui s'observe lui-même et ne cherche pas à s'identifier à une forme maîtresse, trouve autant de différences de soi à soi-même que de soi à autrui. Certes, "si nos faces n'estoient semblables, on ne sçauroit discerner l'homme de la bête ${ }^{11}$ ", ce qui signifie que la ressemblance spécifique n'est pas annulée. Mais elle ne constitue pas pour autant une identité susceptible d'être énoncée selon des critères déterminés : $\mathrm{Si}$ « tous nos jugemens en gros sont lâches et imparfaicts ${ }^{12}$ ", c'est parce que ce qui porte sur le général, et a fortiori sur l'universel, échappe à nos prises.

Ainsi, l'expérience de la singularité, sur laquelle seule Montaigne se prononce, s'inscrit dans une logique de la différence. C'est pourquoi l'essayiste peut compléter sa déclaration de la manière suivante : "si nos faces n'estoient dissemblables, on ne sçauroit discerner l'homme de l'homme ». L'expérience manifeste que " la ressemblance ne faict pas tant un comme la difference faict autre $^{13}$ ", si bien qu'en comparant certains individus, il peut s'avérer qu' "il y a plus de distance de tel homme à tel homme qu'il n'y a de tel homme à telle beste $^{14}{ }$. Autrement dit, parce que l'observation de l'individualité est recension des différences, qu'il s'agisse de soi-même ou d'autrui, il est impossible de se saisir soi-même comme "un ", de connaître son identité, puisque cette connaissance de soi est incompatible avec l'expérience effective de soi comme différence à soi, qui fait qu'on peut même parvenir à se regarder de manière distanciée et avec perplexité " comme un voisin, comme un $\operatorname{arbre}^{15}$ ».

$8 \quad$ Ibid., III, 13, p. 1077. C'est moi qui souligne.

9 III, 9, p. 964.

10 III, 13, p. 1029.

11 III, 13, p. 1070.

12 III, 8, p. 943. C'est moi qui souligne.

13 III, 13, p. 1065.

14 I, 42, p. 258.

15 III, 8, p. 942 : «Je ne m’ayme pas si indiscretement et ne suis si attaché et meslé à moy que je ne me puisse distinguer et considerer à quartier : comme un voisin, comme un arbre. » 
Dans ces conditions, pour quelles raisons ne cessons-nous pas d'aspirer à l'identité ? Pourquoi ne pouvons-nous pas nous passer d'un rapport à nousmêmes qui procède de la reconnaissance de notre identité propre?

\section{Le désir inextinguible d'identité}

Le désir obstiné d'identité qui nous porte à nous constituer une identité par emprunt, au gré de constructions imaginaires successives et d'étayages fictifs de soi, s'explique par le fait que le désir humain n'est pas naturellement ordonné à soi, mais qu'il tend à regarder ailleurs, au-dehors de soi : "Pour ne nous desconforter, nature a rejeté bien à propos l'action de nostre veuë au dehors ${ }^{16}$ ". Nous ne cessons de nous échapper à nous-mêmes, "nous pensons tousjours ailleurs ", " nous ne sommes jamais chez nous, nous sommes tousjours au delà ${ }^{17}$. Mais par cet échappement même, qui nous dérobe à nous-mêmes, nous nous disposons à recevoir satisfaction de l'extérieur, par la reconnaissance qu'autrui est susceptible de nous apporter, par l'image qu'il nous renvoie de nous-mêmes, du dehors.

Au-dedans, le moi est vide ${ }^{18}$, car au départ indéterminé, inconsistant à l'intérieur de lui-même, creux. Il ne s'affirme pas dans son être par un effort pour conserver ou réaliser son essence individuelle, mais comme pouvoir d'exister dans le passage, d'un objet du désir à l'autre, non seulement sous le regard d'autrui, mais par autrui. Le moi ne cesse de se donner une consistance et une constance provisoires, par le lien qu'il entretient avec l'extériorité, précisément parce que ce qu'il est susceptible de devenir n'est pas à rapporter à une forme essentielle, mais à la contingence du monde dans lequel il est jeté et qui est la source de toutes les déterminations dont il se remplit. Le moi ne préexiste donc pas à la société dans laquelle il vit, mais il se constitue à partir d'elle, à partir du réseau de relations dans lequel il est pris et sans lequel il n'est rien, puisque le moi a besoin d'être soutenu dans son être par d'autres que soi, qui lui confèrent une existence nécessairement empruntée : «Il faut jouer deuement nostre rolle, mais comme rolle d'un personnage emprunté ${ }^{19}$ ".

Le fait d'avoir à revêtir des identités sociales n'est pas chez Montaigne (à la différence de chez Rousseau par exemple) à déplorer, au nom d'une inauthenticité du moi, car le moi n'est rien sous les emprunts dont il a besoin pour advenir à lui-même, c'est-à-dire non plus pour réaliser une forme substantielle et singulière qui lui conférerait une identité personnelle, mais pour configurer son existence par l'accoutumance. Car Montaigne entend par accoutumance

16 III, 9, p. 1000.

17 Voir respectivement I, 8 p. 32-33 (la métaphore du cheval échappé), III, 4, p. 834, et I, 3, p. 15.

18 II, 16, p. 618 : « Nous sommes tous creux et vuides ".

19 III, 10, p. 1011. 
non pas tant les coutumes déjà constituées, qu'une puissance métamorphique (qu'il compare au breuvage de Circée) ou force plastique qui donne forme à notre vie sociale, l'ordonne à des pratiques qui viennent à nous manquer si elles nous font défaut, parce qu'elles diversifient notre nature par l'adoption d'identités d'emprunt auxquelles nous nous attachons ${ }^{20}$.

Le moi en effet est creux plutôt par excès que par défaut de déterminations contradictoires qui le traversent et face auxquelles, rétrospectivement, il se perd, dans la mesure où, accumulées, elles font de lui un écheveau de dissemblances ou discordances impossible à démêler ${ }^{21}$. Il en résulte qu'il lui est plus facile de dresser son autoportrait par retranchement de ce qu'il éprouve ne pas être, de ce à quoi il ne s'identifie pas. Ainsi Montaigne se décrit-il, en tant que Maire de Bordeaux, face à ses administrés, comme " sans memoire, sans vigilance, sans experience, et sans vigueur ; sans haine aussi, sans ambition, sans avarice et sans violence ${ }^{22} »$. Ces déterminations toute négatives ne sauraient le combler, contribuer à la constitution positive de son identité, puisqu'elles ne sont là que pour signifier, dans une déclaration publique, ce que le moi ne peut assumer comme lui étant propre, ce à quoi il ne peut s'engager auprès d'autrui à partir de la manière dont il se sent lui-même, et qu'il ne peut endosser avant d'être entré en fonction.

En revanche, lorsque le moi se voit par le regard d'autrui, il se remplit des déterminations successives (qui proviennent de ses différentes mutations) qui l'authentifient socialement, à partir des fonctions qu'il exerce et dans lesquelles il est susceptible de se reconnaître. Et cela implique l'apprentissage de manières régulières d'être qui procèdent d'une ritualisation de l'existence sur la base de pratiques communes, elles-mêmes rendues possibles par un mimétisme spontané ${ }^{23}$ entre nous et les autres qui repose autant sur la mécanique corporelle que sur la sympathie, au sens humien d'une contagion émotionnelle et affective qui procède des passions et de l'imagination.

Mais la constitution de cette identité à partir des manières de faire que tous partagent ne relève pas pour autant de ce que l'on serait tenté de dénoncer comme un processus d'aliénation sociale, puisque l'identité ne préexiste pas à la vie sociale, et que le « moi » a besoin d'être soutenu dans son être par un autre que soi. Son désir d'identité se réalise par ces relations d'interdépendance, qui certes l'assujettissent à des normes, mais qui en même temps lui donnent la possibilité de s'en affranchir, dès lors qu'il s'est constitué comme sujet autonome à partir de ces liens, et qu'il peut prendre de la distance par rapport à ces formes d'assujettissement auxquelles il s'est attaché (aux deux sens du terme).

\footnotetext{
20 Sur l'accoutumance, voir III, 10, p. 1010 et III, 13, p. 1080.

21 Voir II, 1, p. 335.

22 III, 10, p. 1005.

23 III, 5, p. 875 : «J'ay une complexion singeresse et imitatrice».
} 
En effet, pour Montaigne, les mécanismes sociaux d'identification inculquent une plasticité qui permet à l'individu, une fois le vide « rempli » et le moi constitué, de lâcher du lest, de régler l'adhésion à ces processus d'identification à partir de «l'arrière-boutique ». C'est là que les liens d'identification sont relâchés, sans que ce recul soit à comprendre - comme l'interpréteront Pascal et la tradition libertine - comme une scission entre le dedans (qui chercherait à préserver l'authenticité) et le dehors (aliéné et duplice). En effet, la distance critique à l'égard des formes d'identification sociale n'aurait pas été possible sans cet attachement de l'individu à une extériorité constitutive de son identité singulière, attachement qui lui-même suppose l'expérience de l'échappement à soi, et le désir de devenir soi par autrui.

C'est pourquoi l'individu ne peut pas se passer de la notion d'identité : l'identité accidentelle, qui le configure du dehors, est ce par quoi il s'authentifie socialement, ce par quoi il trouve de quoi satisfaire son désir de devenir soi par autrui, au moyen d'un étayage fictif.

Et cet étaye fictif n'est pas pour autant faux, car même s'il procède de l'imagination, il permet à l'individu de s'accréditer à ses propres yeux et aux yeux des autres, rétrospectivement, par un retour sur ce qu'il dit être devenu, et qu'il accepte d'être.

\section{La reconnaissance mutuelle de l'identité}

Même dans le cas d'une imposture où un homme se fait passer pour un autre, l'usurpation d'identité peut être tellement réussie, et faire l'objet d'une telle reconnaissance publique, qu'il demeure difficile, non seulement de reconnaître l'identité première, mais encore de ne pas garder une préférence pour l'imposteur une fois démasqué, si ce dernier a su mieux jouer son rôle.

C'est le cas d'un contemporain de Montaigne, le stupéfiant Arnaud du Thill, le faux Martin Guerre, qui comme l'analyse Nathalie Zemon Davis dans Le Retour de Martin Guerre, bénéficie toujours (jusqu'à aujourd'hui) de la sympathie du public et auditeurs du récit, premièrement pour avoir mieux joué le rôle de mari, de père, et de propriétaire que le vrai Martin Guerre, deuxièmement pour avoir accédé à l'honnêteté dans l'exercice de son personnage emprunté, comme il ne l'avait jamais été en son nom propre, garçon de mauvaise vie qu'il était ${ }^{24}$. C'est pourquoi la micro-société dans laquelle évolue le faux Martin Guerre continue à l'identifier comme le vrai, comme

24 Dans Le Retour de Martin Guerre, Cambridge, MA, Harvard University Press, 1982, traduction française, Tallandier, 2008, Nathalie Zemon Davis analyse le cas de Martin Guerre comme un phénomène d'identification stupéfiant. Le désir de se forger une identité et de se construire une nouvelle vie est au cour de l'imposture : "Vif ou mort, il [le faux Martin Guerre] défendait contre un étranger [le vrai Martin Guerre] l'identité qu'il s'était forgée », ibid., p. 155. 
méritant d'être celui qu'il prétendait faussement être et qu'il est devenu dans l'imaginaire collectif, ou du moins, en raison du caractère extraordinaire de l'imposture souligné par Montaigne, comme méritant cette clémence liée à un doute qui ne lui a pas été accordé25. L'usurpateur, celui qui s'est forgé une identité de toutes pièces, à la place d'un autre, a mieux joué son rôle social que le vrai, a mieux défendu son identité que le vrai Martin Guerre, devenu étranger aux siens, faute d'avoir su porter à la perfection l'art de se façonner soi-même ${ }^{26}$. Par ses déclarations, par la manière de se peindre avec les couleurs d'un autre, Arnaud du Thill a appris à être fidèle au portrait qu'il voulait dresser de lui-même et est devenu l'homme de bien qu'il n'avait jamais été, d'une manière conforme au processus d'identification de soi théorisé par Montaigne et dont ce dernier fait l'expérience par la publication de ses Essais: " Je sens ce proffit inesperé de la publication de mes meurs, qu'elle me sert aucunement de regle. Il me vient par fois quelque consideration de ne trahir l'histoire de ma vie. Cette publique declaration m'oblige de me tenir en ma route, et à ne desmentir l'image de mes conditions $[\ldots]^{27}$ ".

Cela signifie qu'être soi-même n'est pas autre chose qu'être fidèle au récit de soi par lequel on s'est constitué une identité. En d'autres termes, l'authentification sociale de soi suppose le récit de soi, la confession publique de sa vie, dont le but ordinaire (lorsqu'il ne s'agit pas d'une imposture) est moins de se réformer - car la réforme de soi implique toujours une prédétermination de ce que l'on doit être, et donc une réification de soi - que d'accepter ce que la contingence a fait de nous à partir d'une réappropriation de soi qui procède d'une rétrospection. La rétrospection, en réorganisant dynamiquement des morceaux de vie épars, les lopins dont nous sommes faits, relève aussi de l'invention de soi. Son but est bien la jouissance (" scavoyr jouyr loiallement de son estre "), au sens d'une acceptation de soi qui suppose une situation d'interaction avec autrui, face auquel on ne veut pas montrer autre chose de soi que ce que l'on a déclaré être, et qui nous a engagés à le devenir dans les faits, comme en paroles : «Nous ne sommes hommes, et ne nous tenons les uns aux autres que par la parole ${ }^{28} »$.

25 Voici ce qu'en dit Montaigne dans les Essais en III, 11, p. 1030 : «Je vis en mon enfance un procés, que Corras, conseiller de Toulouse, fist imprimer, d'un accident estrange : de deux hommes qui se presentoient l'un pour l'autre. Il me souvient (...) qu'il me sembla avoir rendu l'imposture de celuy qu'il jugea coulpable si merveilleuse et excedant de si loing nostre connoissance, et la sienne qui estoit juge, que je trouvay beaucoup de hardiesse en l'arrest qui l'avoit condamné à estre pendu ".

26 "Arnaud du Tilh devient une sorte de héros, un Martin Guerre plus réel que l'homme au cœur sec et à la jambe de bois ; la tragédie est moins dans l'imposture que dans sa découverte. ", Nathalie Zemon Davis, ibid., p. 191.

27 Montaigne, Essais, III, 9, p. 980.

28 Essais, I, 9, p. 36. 
Ce retour sur la façon dont chacun prétend qu'il « s'est tenu dans le passage " ne concerne donc pas seulement un imaginaire social (qui expose à être victime d'une supercherie). Il implique aussi un consensus social, qui lui-même présuppose l'exercice du jugement, ainsi qu'un engagement mutuel des hommes par la parole qui ne serait pas possible sans l'existence d'une communauté linguistique d'appartenance. Comme Carlo Ginzburg l'a souligné, en se référant aux travaux de Jakobson, la conscience de soi n'est possible que si elle s'éprouve par contraste, en tant que « je " qui s'adresse à un " tu ", sachant que " je » devient " tu " par celui qui dit « je ». Parce que l'identité individuelle se conquiert par la parole, la rétrospection constitutive de l'identité renvoie à la manière dont chacun fait un retour narratif sur sa position, se dote d'un contenu énonçable qui donne consistance à celui qui dit " je " et qui se modèle par le discours sur soi. Ainsi, on ne peut se passer de l'acquiescement des autres, car lorsque les autres à leur tour disent " je " en leur nom propre, ils accréditent le discours entendu, tout en désirant en retour bénéficier d'une forme de reconnaissance explicitement exprimée. Et c'est par cette reconnaissance que le sujet réalise son désir d'identité auquel il aspirait, comblant une forme vide, ou si l'on préfère actualisant des possibles à partir des projections symboliques véhiculées par le langage.

Le problème est que cette reconnaissance mutuelle ne va pas de soi, car le désir d'être reconnu dans son identité par l'autre est ambivalent : si le rapprochement désiré conduit certains hommes à s'identifier mutuellement par des pratiques sociales, il les conduit aussi en même temps à refuser à d'autres hommes cette reconnaissance. Le désir d'identification mutuelle non seulement n'exclut pas le conflit, mais l'implique, car le désir qui nous pousse au-dehors pour nous " remplir» trouve aussi de quoi s'assouvir dans la destruction et la dévoration de celui qui n'a pas été reconnu mais a été au contraire rejeté en dehors de l'humanité. Désir d'identification auprès d'autrui et désir de destruction d'autrui vont de pair : le patriotisme (ou sentiment fervent qu'un peuple éprouve pour lui-même) est d'autant plus fort que les pulsions de destruction et de cruauté (qui portent les hommes à refuser de reconnaître d'autres hommes comme leurs semblables) sont redirigées à l'extérieur de la communauté vers un autre peuple considéré comme barbare. D'une manière plus insidieuse, le plaisir qu'éprouvent deux compatriotes français de se rencontrer par hasard "en Hongrie " assouvit selon Montaigne la même pulsion xénophobe : le sentiment vif d'une identité nationale commune qui rapproche les individus trouve sa source dans une hostilité latente à l'égard des étrangers ${ }^{29}$.

29 Voir III, 9, p. 985 : « Retrouvent ils un compatriote en Hongrie, ils festoyent cette avanture : les voylà à se ralier et à se recoudre ensemble, à condamner tant de meurs barbares qu'ils voient. Pourquoy non barbares, puis qu'elles ne sont françoises?" 
Pour cette raison, Montaigne n'érige jamais en modèle des formes sociales d'identification qui tendraient à la fusion communautaire et du même coup au rejet de la conflictualité au-dehors. Lorsque les membres d'un peuple se pensent comme faisant partie d'un seul corps digne d'un amour tel qu'on pourrait se sacrifier pour lui, les instincts à l'inhumanité que l'on trouve en chaque peuple se déverse massivement à l'extérieur de la communauté sur d'autres populations qui ne seront jamais reconnues dans leurs différences, mais niées dans leurs différences, et massacrées, anéanties en raison de leurs différences.

De manière symétrique, lorsque des individus s'identifient à la communauté à laquelle ils appartiennent, le rapprochement entre les membres se fait sans distance, et les différences sociales étant annulées, la relation sociale à partir de laquelle l'individu pouvait advenir à lui-même, dans son identité singulière, est supprimée. Parce que la constitution de l'identité individuelle est toujours à rapporter à l'extériorité, à la contingence d'une existence sociale, qui en est la condition, elle ne peut advenir si l'individu est intégré à la société dans laquelle il se trouve sur la base d'un don de soi à la communauté qui supprime la différence entre le dedans et le dehors, entre lui et les autres. Dans ce schéma, l'individu est anéanti par l'identification sociale qu'on lui impose, si bien qu'il ne peut plus apprendre à "être à soi », et encore moins espérer "se donner à autrui sans s'ôter à soi ». Ces expressions montaniennes excluent de s'adonner avec dévotion à l'altérité en cherchant une identité communautaire où le moi, confondu avec sa fonction sociale, se dissoudrait dans le tout, ne s'autorisant à être que sur la base du discours de l'institution. Il ne s'agit jamais de se considérer comme membre d'un corps social n'ayant qu'une seule pensée régie par un principe unificateur spirituel, de souscrire à un discours qui inciterait l'individu à se reconnaître dans une parole dominante. Au contraire, chacun doit pouvoir valider son adhésion à la communauté dont il émane, estimer ce qu'il lui doit et ce qu'il se doit, compte tenu de ce qu'elle vaut.

Si l'identité de l'individu procède donc fondamentalement de son inscription dans une société qui a donné forme à sa vie, elle doit selon Montaigne pouvoir faire l'objet d'une reprise permanente dans un discours ayant le courage de son dire, c'est-à-dire un discours susceptible de contester les formes de domination sociale, de résister à l'abolition des différences individuelles, de se soustraire à l'obligation de penser et de parler comme les autres, par le rejet de l'usage lénifiant de la parole.

\section{Conclusion}

Si nous continuons à avoir soif d'identité, et nous passons difficilement de cette notion, alors même que "nous n'avons aucune communication à l'estre ${ }^{30}$ ", c'est-à-dire que nous sommes par nature dépourvus d'identité, 
c'est certainement parce que l'estime de soi repose en majeure partie sur la constitution sociale d'une position stable à laquelle nous nous identifions. Les Essais de Montaigne nous aident à comprendre ce désir obstiné d'identité, mais sans nous dispenser de porter un regard critique, non complaisant, sur les différents usages de cette notion et sur les risques de déshumanisation que le désir d'identité fait encourir à l'individu comme à la collectivité.

\author{
Sylvia Giocanti \\ UMR 5317 (Cerphi) \\ Université Jean Jaurès, Toulouse \\ sylvia.giocanti@univ-tlse2.fr
}

\title{
Résumé
}

Selon Montaigne, parce que l'unité du moi n'est pas donnée, la manière familière d'être à soi relève de la différence à soi. Le désir d'identité s'articule alors avec le besoin social de se doter de déterminations stables. Les Essais analysent ce processus rétrospectif et discursif comme source d'authentification, et pas nécessairement d'aliénation, dans la mesure où l'individu est conduit à l'acceptation de soi, et où il peut réfléchir de manière critique à son inscription dans la communauté à laquelle il appartient.

\section{Mots-clés}

Montaigne, Martin Guerre, identité, aliénation, reconnaissance.

\section{Abstract}

According to Montaigne, since the unity of the self is not a datum, the ordinary way of beeing oneself comes within the division of the self. Then, desire of identity depends on the social need to provide oneself with permanent determinations. The Essays analyse this retrospective and discursive process as a root of authentification more than alienation, in so far as the individual is led to accept oneself and may critically think about his social integration.

\section{Keywords}

Montaigne, Martin Guerre, identity, alienation, acknowledgement. 